\title{
Hyperoside attenuates dextran sulfate sodium-induced colitis in mice possibly via activation of the Nrf2 signalling pathway
}

\author{
Lei Yang, Lei Shen", Yue Li, Yanxia Li, Shijie Yu and Shanshan Wang
}

\begin{abstract}
Background: Hyperoside (Hyp) is a flavonoid glycoside compound that has been demonstrated to have antiinflammatory, anti-apoptotic and antioxidant effects. However, the impact of Hyp on inflammatory bowel disease (IBD) has not been previously explored. Thus, we evaluated the role of Hyp in dextran sodium sulfate (DSS)-induced acute colitis in mice.

Methods: We established a mouse model of experimental acute colitis by treating mice with drinking water supplemented with 3.0\% DSS for 7 days. The disease activity index (DAI), colon length, histological features and colonic malondialdehyde (MDA) levels were examined using appropriate methods, and COX-2 expression was examined by immunohistochemistry. TNF-a, IL-4, IL-6, IL-10, NF-kB p65, BCl-2, Bax, Caspase-3, nuclear factorerythroid 2-related factor 2 (Nrf2), hemeoxygenase-1 (HO-1) and superoxide dismutase (SOD) levels in colorectal tissues were detected by RT-PCR and western blotting.
\end{abstract}

Results: Hyp significantly attenuated DSS-induced changes in the DAI as well as DSS-induced colonic shortening and histological changes. Hyp also inhibited inflammation, a change reflected by decreases in TNF-a, IL-6, COX-2 and NF-KB p65 expression and increases in IL-10 expression. Hyp suppressed increases in the levels of apoptosisrelated proteins, such as Caspase-3 and Bax, but upregulated the level of the anti-apoptotic protein Bcl2. In addition, Hyp also exerted antioxidant effects. The MDA content was decreased, and the expression of Nrf2 and its downstream targets HO-1 and SOD were increased by Hyp.

Conclusions: Based on these findings, Hyp possesses the ability to attenuate colitis, possibly by mitigating colonic inflammation and apoptosis via activation of the Nrf2 signaling pathway.

Keywords: Hyperoside, DSS-induced colitis, Colonic inflammation, Apoptosis, Nrf2

\section{Background}

Ulcerative colitis (UC), one of the subtypes of inflammatory bowel disease (IBD), is a chronic relapsing and nonspecific inflammatory disorder of the gastrointestinal tract. The prevalence of UC is increasing, and UC has serious effects on the health and quality of life of affected patients. Despite improvements in our understanding of UC in recent years, the etiology and pathogenesis of the disease remain unclear and may be associated with several factors. UC is thought to be

\footnotetext{
* Correspondence: szggyx2017@163.com
Department of Gastroenterology, Key Laboratory of Hubei Province for
Digestive System Disease, Renmin Hospital of Wuhan University, Wuhan

* Correspondence: szggyx2017@163.com Digestive System Disease, Renmin Hospital of Wuhan University, Wuhan 430060, China 430060, China
}

associated with factors such as genetic susceptibility, imbalances between gastrointestinal probiotics and pathogenic bacteria, intestinal mucosa damage, immune dysfunction and other phenomena. Immune dysregulation likely underlies the basic pathogenesis of IBD [1]. The activation of the NF- $\kappa$ B pathway as well as the release of related inflammatory cytokines and proinflammatory mediators, plays an important role in the of UC [2,3], and increases in the frequency tinal epithelial cells, also participate in IBD progression [4]. Furthermore, the roles of oxidative stress and the oxidant/antioxidant balance in UC development have recently received increasing attention [5]. 
Nuclear factor-erythroid 2-related factor 2 (Nrf2), a redox-sensitive transcription factor, is one of the members of the Cap-N-Collar family of transcription factors. Nfr2 features a leucine zipper structure [6] and plays a key role in the antioxidant response. When cells are exposed to oxidative stress, Nrf2 is released from Kelchlike ECH-associated protein 1 (Keap1), which sequesters Nrf2 in the cytoplasm, and then binds to Maf or Jun to form a heterodimer in the nucleus. The heterodimer combines with the antioxidant response element (ARE) to promote the expression of genes encoding many phase II detoxification and antioxidant enzymes, including hemeoxygenase-1 (HO-1), superoxide dismutase (SOD), and NOQ1, thereby improving the ability of the cell to remove electrophilic and reactive oxygen species (ROS) [7]. A Nrf2 deficiency has been shown to exacerbate colonic injury in a mouse model of experimental colitis [8-10], whereas pharmacological activation of Nrf2 produced protective effects on the colon $[10,11]$. The Nrf2 pathway not only upregulates the expression of various antioxidant enzymes but also downregulates a series of inflammatory mediators and attenuates apoptosis $[12,13]$. These Nrf2-mediated processes are vital to the ability of the body to resist different types of injury.

Hyperoside (Hyp, a quercetin-3-O- $\beta$-D-pyran galactose glucoside) belongs to the flavonol glycoside family and is extracted from Hypericum, Ericaceae, Celastraceae and other species. Hyp exerts a wide variety of pharmacological effects, including anti-inflammatory, antioxidant, antitumor, analgesic, and immunoregulatory effects. Several studies have reported the anti-inflammatory and antioxidant properties of Hyp. As shown in the studies by $\mathrm{Li}$ $\mathrm{Y}$ et al. $[14,15]$, Hyp inhibits the activation of NF- $\mathrm{kB}$ and suppresses the secretion of proinflammatory cytokines, including TNF- $\alpha$, COX-2 and iNOS. Based on the results from in vivo and in vitro studies, Hyp enhances the expression of genes encoding many phase II detoxifiers and antioxidants by activating Nrf2 $[15,16]$. In addition, Li ZL et al. [17] showed that Hyp exerted protective effects on $\mathrm{H}_{2} \mathrm{O}_{2}$-induced human umbilical vein endothelial cell lesions by attenuating endothelial cell apoptosis.

In the present study, we attempted to determine whether Hyp-mediated Nrf2 upregulation exerts antiinflammatory and anti-apoptotic effects on DSS-induced $\mathrm{UC}$ in a $\mathrm{C} 57 \mathrm{BL} / 6$ mouse model.

\section{Methods}

\section{Animals}

We obtained 6- to 8-week-old adult male C57BL/6 mice from Beijing HFK Bioscience Co., Ltd. (Beijing, China). All mice were housed in a room with a controlled temperature $\left(21 \pm 2^{\circ} \mathrm{C}\right)$ and humidity $(50 \pm 5 \%)$ and were maintained on a 12-h light/dark cycle. The mice had access to a standard mouse diet and sterile water ad libitum and were acclimated to the laboratory conditions for 7 days before undergoing any experimental procedures. All experimental procedures were conducted in accordance with the institutional guidelines for the care and use of laboratory animals of Renmin Hospital of Wuhan University, Wuhan, China, and conformed to the regulations outlined in the National Institutes of Health Guide for Care and Use of Laboratory Animals.

\section{Reagents}

Hyp (purity $>98 \%$ ) was obtained from Shanghai Yuanye Biotechnology Co., Ltd. (Shanghai, China). DSS (MW 36,000-50,000 Da) was obtained from MP Biomedical (Aurora, OH, USA). The malondialdehyde (MDA) reagent kit was purchased from the Nanjing Jiancheng Bioengineering Institute (Nanjing, China). The DAB kit was purchased from Beijing Solarbio Science \& Technology Co., Ltd. (Beijing, China). Antibodies against COX-2 and Nrf2 were obtained from Santa Cruz Biotechnology Inc. (CA, USA), and antibodies against NF-kB p65, Bcl-2, Bax, and Caspase-3 were obtained from Cell Signaling Technology, Inc. (Beverly, MA, USA). $\beta$-Actin and Lamin B antibodies were purchased from Abcam (Cambridge, UK).

\section{Experimental design}

Twenty-eight mice were randomly allocated into the following four groups (7 mice per group): a control group, a DSS model group, and DSS+Hyp (treated with 80 and $120 \mathrm{mg} / \mathrm{kg}$ Hyp, respectively) groups. Hyp was suspended in $0.5 \%$ carboxymethyl cellulose (CMC). The mice in the control group received normal drinking water and $0.5 \%$ CMC by gavage in the morning for 14 days. The mice in the DSS model group received $0.5 \%$ CMC by gavage for 7 days prior to 3\% DSS (MP Biomedical. 160110) exposure and were then maintained until sacrifice, whereas the mice in the DSS+Hyp (80 and $120 \mathrm{mg} / \mathrm{kg}$, respectively) groups received Нyp suspended in $0.5 \%$ CMC. The body weight, stool consistency, and gross bleeding scores of each animal were recorded to calculate the DAI [18]. The criteria with which the DAI was graded are shown in Table 1. On the morning of day 15, all mice were sacrificed, and the total colon length was measured. The dissected

\begin{tabular}{llll}
\multicolumn{4}{c}{ Table $\mathbf{1}$ The grading rule of disease activity index (DAI) } \\
\hline Score & Weight loss (\%) & Stool consistency & Blood in stool \\
\hline 0 & None & $0=$ normal & $0=$ negative \\
1 & $1-5 \%$ & $2=$ loose stool & $2=$ Hemoccult positive \\
2 & $6-10 \%$ & $4=$ diarrhea & $4=$ gross bleeding \\
3 & $11-20 \%$ & & \\
4 & $>20 \%$ & \\
\hline
\end{tabular}


colon was washed gently with PBS. A portion of the distal colon was subsequently fixed with $10 \%$ buffered formalin for $24 \mathrm{~h}$, and the remaining tissue was immediately frozen in liquid nitrogen and stored at $-80^{\circ} \mathrm{C}$ until use in subsequent experiments.

\section{Histopathology analyses}

A portion of the distal colon, which was fixed with $10 \%$ buffered formalin for more than $24 \mathrm{~h}$, was mounted in paraffin and stained with hematoxylin and eosin before observation under a light microscope. The following previously described histological scoring system [19] was used to grade the severity of the tissue damage induced by DSS: percent tissue damage: $0=$ no tissue damage, $1=1-25 \%, 2=26-50 \%, 3=51-75 \%$ and $4=76-100 \%$; extent of tissue damage: $1=$ mucosa, $2=$ mucosa and submucosa and $3=$ beyond the submucosa; extent of crypt damage: $1=$ basal $1 / 3$ damaged, $2=$ basal $2 / 3$ damaged, $3=$ only the surface epithelium was intact and $4=$ the entire crypt and epithelium were lost; and degree of inflammation: 1 =slight, $2=$ moderate and $3=$ severe.

\section{Immunohistochemistry analyses}

A portion of the colon, which was fixed with $10 \%$ buffered formalin and then dehydrated in a graded ethanol series, was mounted in paraffin and then sectioned (4 $\mu \mathrm{m})$. The sections were dewaxed and rehydrated before being incubated with $3 \% \mathrm{H}_{2} \mathrm{O}_{2}$. Sections were then incubated with a COX-2 antibody (dilution 1:50) in a humidified chamber overnight at $4^{\circ} \mathrm{C}$. Sections were washed and then incubated with the appropriate secondary antibodies and streptavidin-horseradish peroxidase (HRP) for $30 \mathrm{~min}$ at room temperature. Each section was treated with freshly prepared diaminobenzidine (DAB) before being counterstained with hematoxylin. Images were obtained and observed under a microscope.

\section{Malondialdehyde (MDA) analyses}

The MDA content was determined with a commercial kit that assesses MDA levels through a process dependent on thiobarbituric acid (TBA) reactivity, according to the manufacturer's protocol. We added TBA to a protein-free supernatant, which was obtained by centrifuging colonic tissue homogenates with trichloroacetic acid. The TBA was allowed to react with the supernatant for $60 \mathrm{~min}$ at $95^{\circ} \mathrm{C}$ before the mixture was cooled rapidly. The absorbance of the supernatant was assessed at $532 \mathrm{~nm}$ using a spectrophotometer.

\section{Real-time PCR}

Total RNA was isolated from the colonic tissue samples using TRIzol reagent (Invitrogen, Carlsbad, CA), according to the manufacturer's instructions and was verified by determining the absorbance at $260 / 280 \mathrm{~nm}$ using a spectrophotometer. The RNA was then reverse-transcribed into cDNA with reverse transcriptase (GeneCopoeia, Rockville, USA). Real-time PCR (RT-PCR) was conducted with SYBR Green Master Mix (Vazyme, Nanjing, China). $\beta$-Actin, a housekeeping gene, was used as a positive control. The sequences of all primers used for the RT-PCR experiments are presented in Table 2.

\section{Cytosolic and nuclear protein extraction and western blotting}

Total cytosolic and nuclear proteins were extracted from the colonic tissue samples using the appropriate kits, according to the manufacturer's instructions (Thermo Fisher Scientific Inc., MA, USA). Protein concentrations were determined with a BCA Protein Assay Kit (Beyotime, Shanghai, China), and the proteins were boiled for $5 \mathrm{~min}$. The proteins $(40 \mu \mathrm{g})$ were then separated by SDS-PAGE before being transferred to polyvinylidene difluoride (PVDF) membranes (Millipore, CA, USA). After blocking with 5\% skim milk in TBST for at least $2 \mathrm{~h}$ at room temperature, membranes were incubated with primary antibodies against the following proteins overnight at $4^{\circ} \mathrm{C}$ : NF-kB p65, Bcl-2, Bax, Caspase-3 and Nrf2. LaminB and $\beta$-actin were used as loading controls for the nuclear and

Table 2 Primer sequences for real-time PCR

\begin{tabular}{|c|c|c|}
\hline Name & Primer & Sequence \\
\hline \multirow[t]{2}{*}{$\beta$ - actin } & Forward & 5'- CACGATGGAGGGGCCGGACTCATC-3' \\
\hline & Reverse & 5'- TAAAGACCTCTATGCCAACACAGT-3' \\
\hline \multirow[t]{2}{*}{ Nrf2 } & Forward & 5'- GAGCTAGATAGTGCCCCTGG -3' \\
\hline & Reverse & 5'- CAGGACTCACGGGAACTTCT -3' \\
\hline \multirow[t]{2}{*}{ SOD } & Forward & 5'- AACCATCCACTTCGAGCAGA -3' \\
\hline & Reverse & 5'- GGTCTCCAACATGCCTCTCT -3' \\
\hline \multirow[t]{2}{*}{$\mathrm{HO}-1$} & Reverse & 5'- CCCTAATGAGCTGCCCTACA -3' \\
\hline & Forward & 5'- TTCATCCCCACTCTCTTCGG -3' \\
\hline \multirow[t]{2}{*}{ TNF-a } & Forward & $5^{\prime}-$ ACCCTCACACTCACAAACCA - $3^{\prime}$ \\
\hline & Reverse & 5'- GGCAGAGAGGAGGTTGACTT -3' \\
\hline \multirow[t]{2}{*}{$\mid \mathrm{L}-4$} & Forward & 5'- TCTCGAATGTACCAGGAGCC-3' \\
\hline & Reverse & 5'- ACCTTGGAAGCCCTACAGAC-3' \\
\hline \multirow[t]{2}{*}{ IL-6 } & Forward & 5'-GTTGCCTTCTTGGGACTGAT-3' \\
\hline & Reverse & 5'-ATTAAGCCTCCGACTTGTGA-3' \\
\hline \multirow[t]{2}{*}{ IL-10 } & Forward & 5'- ATAACTGCACCCACTTCCCA-3' \\
\hline & Reverse & 5'- GGGCATCACTTCTACCAGGT-3' \\
\hline \multirow[t]{2}{*}{$\mathrm{BCl}-2$} & Forward & 5'- TCGCAGAGATGTCCAGTCAG-3' \\
\hline & Reverse & 5'- ATCTCCCTGTTGACGCTCTC-3' \\
\hline \multirow[t]{2}{*}{ Bax } & Forward & 5'-TCATGAAGACAGGGGCCTTT -3' \\
\hline & Reverse & 5'-GTCCACGTCAGCAATCATCC - $3^{\prime}$ \\
\hline \multirow[t]{2}{*}{ Caspase-3 } & Forward & 5'- CTACAGCACCTGGTTACTATTC-3' \\
\hline & Reverse & 5'- TACAGTTCTTTCGTGAGCAT-3' \\
\hline
\end{tabular}


total or cytoplasmic protein fractions, respectively. The membranes were washed at least three times before incubation with the appropriate HRP-conjugated secondary antibodies. Protein bands were exposed to film and then quantified using ImageJ software (NIH, Bethesda, MD).

\section{Statistical analyses}

The data obtained from the experiments described above were analyzed using SPSS 16.0 software and are presented as means \pm S.D. One-way analysis of variance (ANOVA) and Student's t tests were used to assess the differences among and between groups, respectively. $P$ values $<0.05$ were considered statistically significant.

\section{Results}

\section{Effects of Hyp on the DAI and colon length}

As shown in Fig. 1b, the DAI (as determined by body weight loss, Fig. 1a; stool consistency and bloody stool scores) was increased in DSS-induced mice compared with healthy control mice in the present study. Hyptreated mice had a lower DAI than the DSS model group, and the difference in the DAI between the two Hyp-treated groups was statistically significant. As shown in Fig. 1c, the colon length was reduced in DSSinduced mice compared with control mice; however, the Hyp treatment attenuated this effect on the Hyp-treated groups. Thus, Hyp alleviates the symptoms of colitis in the mouse model of DSS-induced colitis.

\section{Hyp ameliorates histological damage}

The mice in DSS model group displayed abnormalities in the colonic architecture, such as mucosal ulcerations, glandular defects and inflammatory cell infiltration, whereas the mice in the healthy control group displayed a normal colonic structure. The Hyp treatment ameliorated these histological changes (Fig. 2). Hyp administration also decreased the histological scores in the Hyptreated groups compared with the DSS model group (Fig. 2), indicating that Hyp exerts significant protective effects on inflammation-induced intestinal damage in mice with DSS-induced colitis.

\section{Effects of Hyp on colonic inflammation}

Inflammation is a major factor during UC progression. Based on the western blotting data, NF-kB p65 was expressed at lower levels in the Hyp-treated groups than in the DSS model group (Fig. 3a and b). Levels of TNF$\alpha$, IL-4 and IL-6 mRNA were significantly increased in the DSS model group compared with the healthy control group, indicating that DSS significantly increased intestinal inflammation in the DSS-induced colitis mouse model. However, Hyp decreased the expression of TNF$\alpha$ and IL- 6 mRNA in the colon. However, a significant difference in the expression of IL-4 mRNA was not observed between the DSS- and Hyp-treated groups. IL-10 mRNA was expressed at higher levels in the Hyp-treated groups than in the DSS model group (Fig. 3c). Moreover, COX-2 expression was decreased by treatment with Hyp in the Hyp-treated groups compared with the DSS model group (Fig. 4).

\section{Effects of Hyp on apoptosis}

The expression levels of Caspase-3, Bax and Bcl-2 were quantified by western blotting. Levels of Caspase- 3 and Bax protein in the colon were significantly increased in the DSS-induced group compared with the healthy control group, and Bcl-2 protein was expressed at significantly lower levels in DSS-induced mice than in healthy

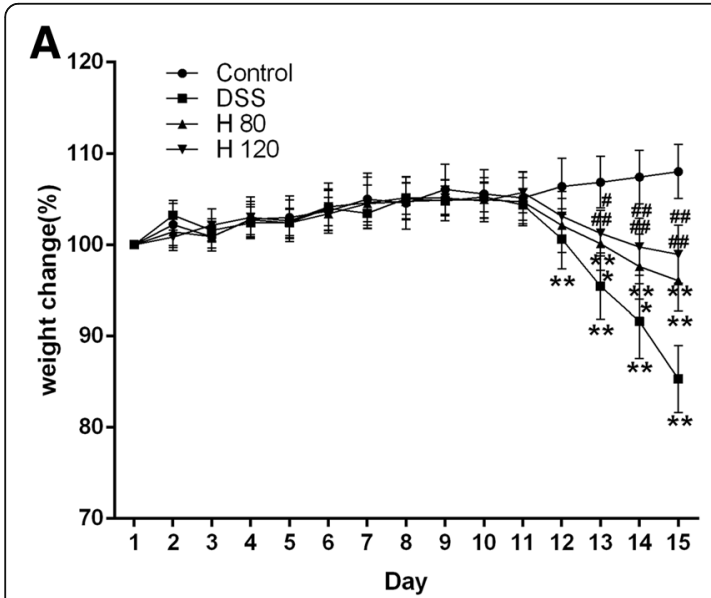

\section{B}

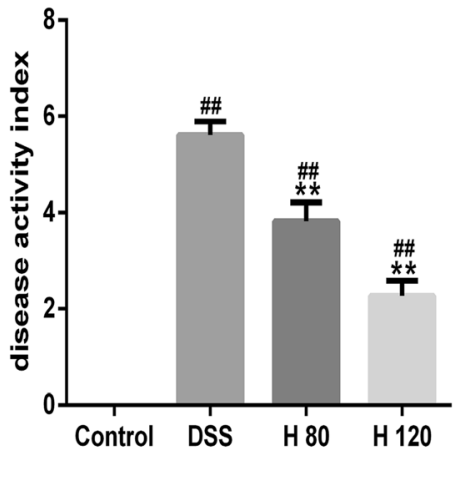

C

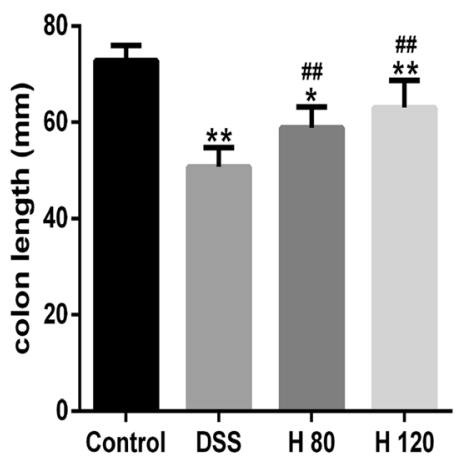

Fig. 1 Protective role of Hyp against DSS-induced acute colitis in mice. a Body weight changes were measured. $\mathbf{b}$ Disease activity index (DAI) was detected. c Statistics of colorectum length of each group were recorded. H 80 and H 120, mice treated with DSS and Hyp 80 and 120 mg/kg respectively. Data are presented as mean \pm S.D. $(n=7)$. Significance: ${ }^{*} P<0.05$, ${ }^{*} P<0.01$ in comparison with DSS model group; ${ }^{\#} P<0.05$, \#\# $P<0.01$ in comparison with control group 


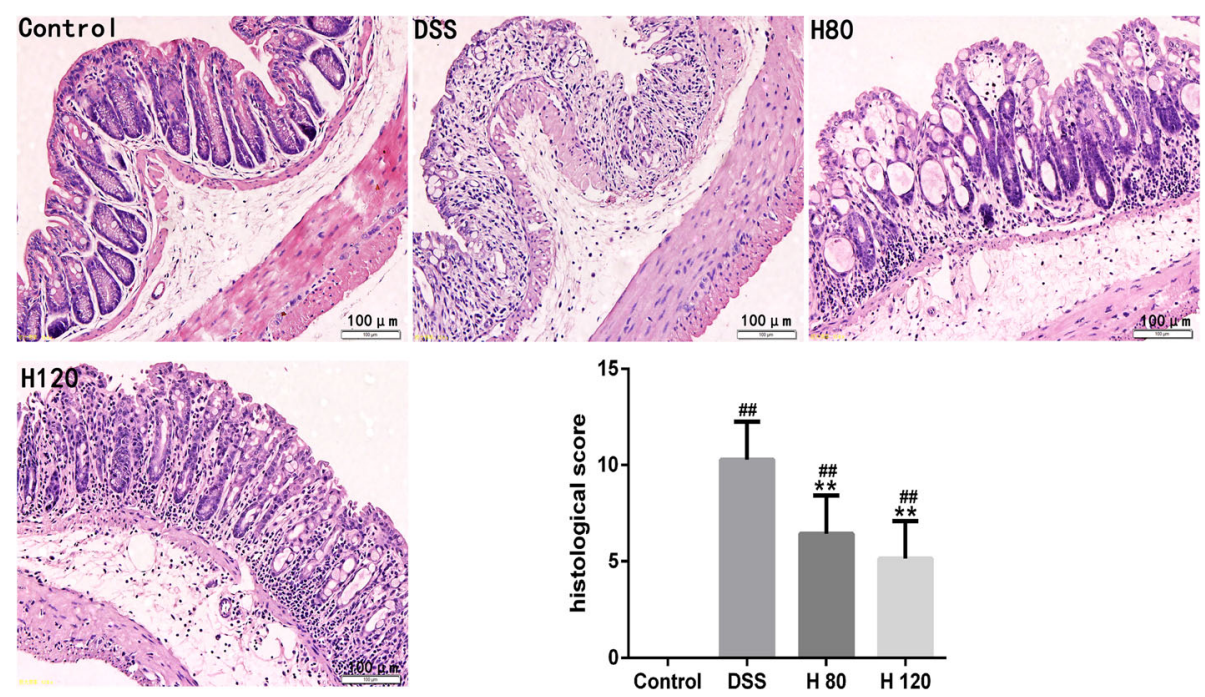

Fig. 2 Hyp alleviated DSS-induced colon damage in mice. Representative H\&E-stained colorectum sections(magnification 200) and histology score. H 80 and H 120, mice treated with DSS and Hyp 80 and $120 \mathrm{mg} / \mathrm{kg}$ respectively. Data are presented as mean \pm S.D. $(n=7)$. Significance: ${ }^{*} P<0.05$, ${ }^{*} P<0.01$ in comparison with DSS model group; ${ }^{\#} P<0.05$, ${ }^{\# \#} P<0.01$ in comparison with control group

control mice (Fig. 5a and b), indicating that the rate of colonic apoptosis in mice with DSS-induced colitis was increased compared with healthy control mice. Hyp increased the protein expression of Bcl-2 and decreased the protein expression of Caspase- 3 and Bax in the Hyp- treated group compared with the DSS model group (Fig. $5 \mathrm{a}$ and b). Changes in the levels of Bcl-2, Bax and Caspase-3 mRNA, quantified by RT-PCR, were consistent with changes in the protein levels of Bcl-2, Bax and Caspase-3 (Fig. 5c).

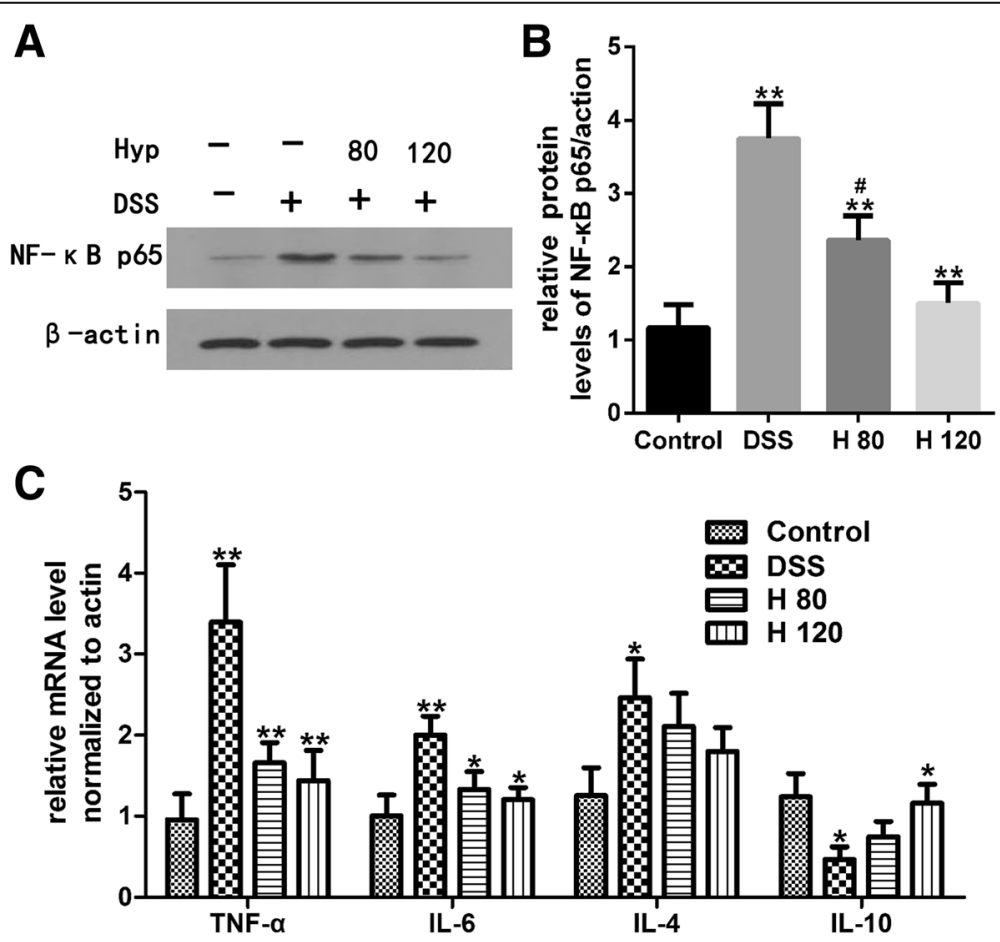

Fig. 3 Hyp reduced colonic levels of NF-KB p65,TNF-a, IL-6 and enhanced the levels of IL-10 in DSS-induced mice. a The expressions of NF-KB p65 via western blotting. b The relative protein expressions of NF-KB p65 were normalized to $\beta$ - actin. $\mathbf{c}$ Gene expression of TNF-a, IL-6, IL-4, IL-10 was used to detected via RT-PCR. H 80 and H 120, mice treated with DSS and Hyp 80 and $120 \mathrm{mg} / \mathrm{kg}$ respectively. Data are presented as mean \pm S.D. $(n=7)$. Significance: ${ }^{*} P<0.05$, ${ }^{*} P<0.01$ in comparison with DSS model group. ${ }^{\#} P<0.05,{ }^{\#} P<0.01$ in comparison with control group 


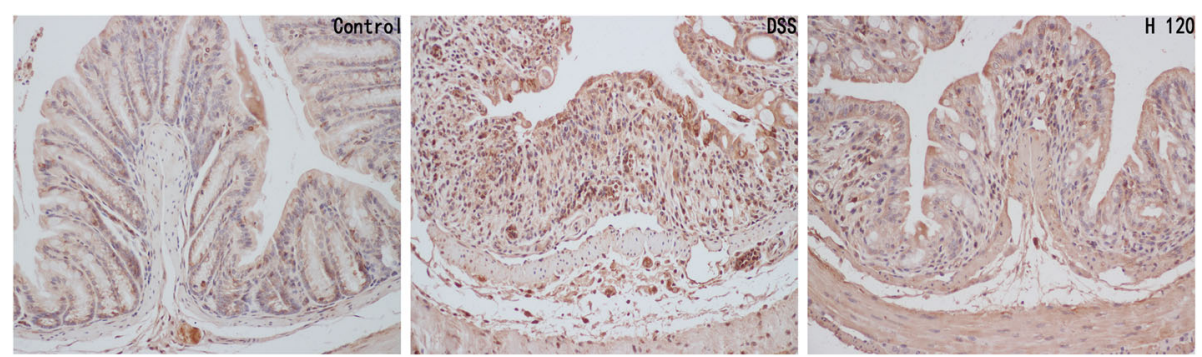

Fig. 4 Hyp alleviated the colonic expression of COX2. Immunohistochemical analysis of COX2 (magnification, 200X). H 80 and H 120, mice treated with DSS and Hyp 80 and $120 \mathrm{mg} / \mathrm{kg}$ respectively. Data are presented as mean \pm S.D. $(n=7)$. Significance: ${ }^{*} P<0.05$, ${ }^{* *} P<0.01$ in comparison with DSS model group; ${ }^{\#} P<0.05,{ }^{\# \#} P<0.01$ in comparison with control group

\section{Hyp decreases the colonic MDA content}

MDA, an important byproduct of lipid peroxidation, is an important index of oxidation reactions in the cell. The MDA content was significantly increased in the DSS model group compared with the healthy control group. However, the MDA content was dramatically decreased in the Hyp-treated groups compared with the DSS model group (Fig. 6a).

\section{Effects of Hyp on the Nrf2 signaling pathway}

The cytosolic and nuclear levels of the Nrf2 protein were significantly reduced in DSS-induced mice compared with healthy control mice (Fig. 6b and c). Levels of Nrf2,
HO-1 and SOD mRNA were detected using RT-PCR, and the levels of HO-1 and SOD mRNA were significantly lower in the colons of DSS-induced mice than in healthy control mice. The Hyp treatment markedly increased the levels of Nrf2, HO-1 and SOD mRNA in DSS-induced mice compared with healthy control mice (Fig. 6d). Based on these findings, Hyp exerted its beneficial effects on DSS-induced colitis through the Nrf2 signaling pathway.

\section{Discussion}

$\mathrm{UC}$ is a chronic relapsing intestinal inflammatory disease that is detrimental to the physical and mental health of
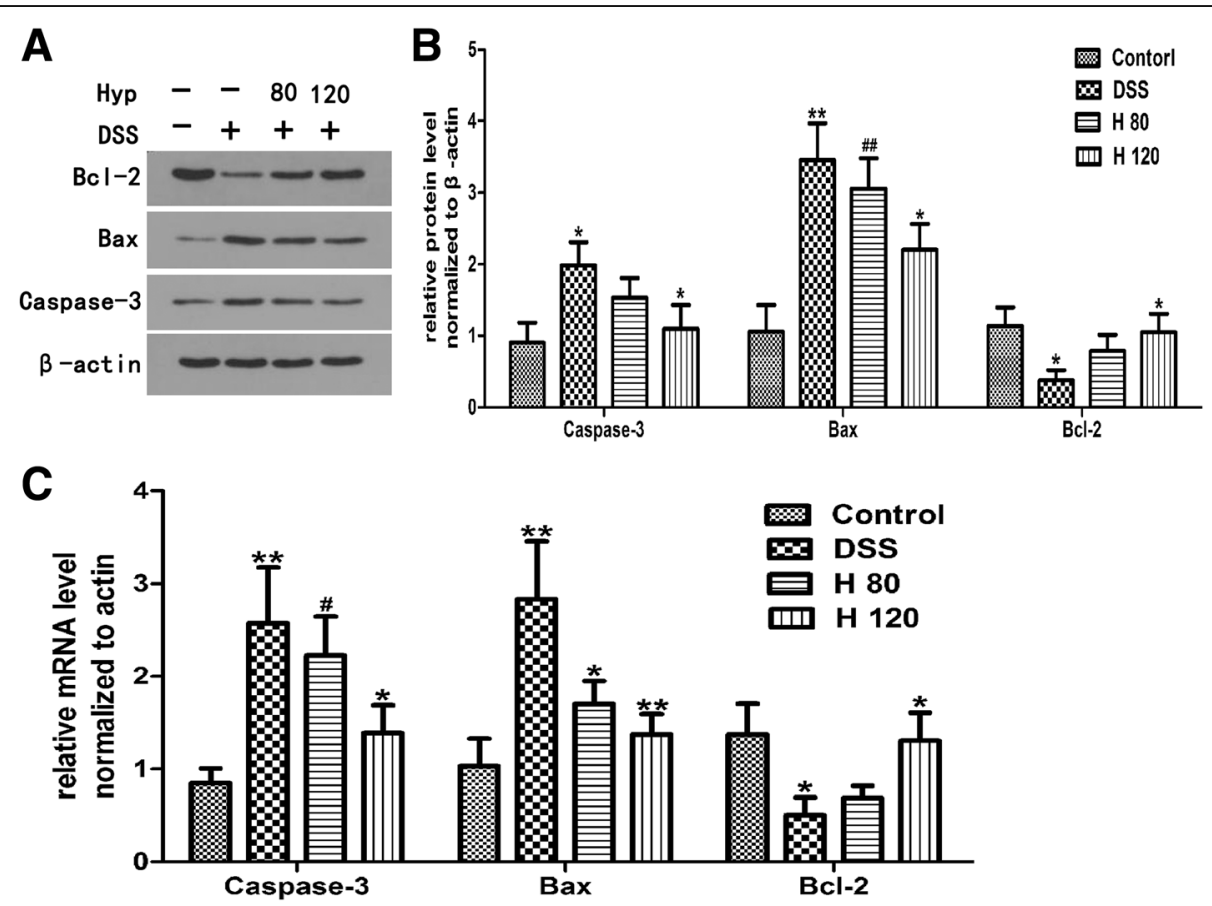

Fig. 5 The effects of Hyp on apoptosis. a The expressions of Caspase-3, Bax and BCl-2 via western blotting. $\mathbf{b}$ The relative protein expressions of Caspase-3, Bax and Bcl-2 were normalized to $\beta$ - actin. c Gene expression of Caspase-3, Bax and Bcl-2 via RT-PCR. H 80 and H 120, mice treated with DSS and Hyp 80 and $120 \mathrm{mg} / \mathrm{kg}$ respectively. Data are presented as mean \pm S.D. $(n=7)$. Significance: ${ }^{*} P<0.05,{ }^{* *} P<0.01$ in comparison with DSS model group; ${ }^{\#} P<0.05,{ }^{\# \#} P<0.01$ in comparison with control group 

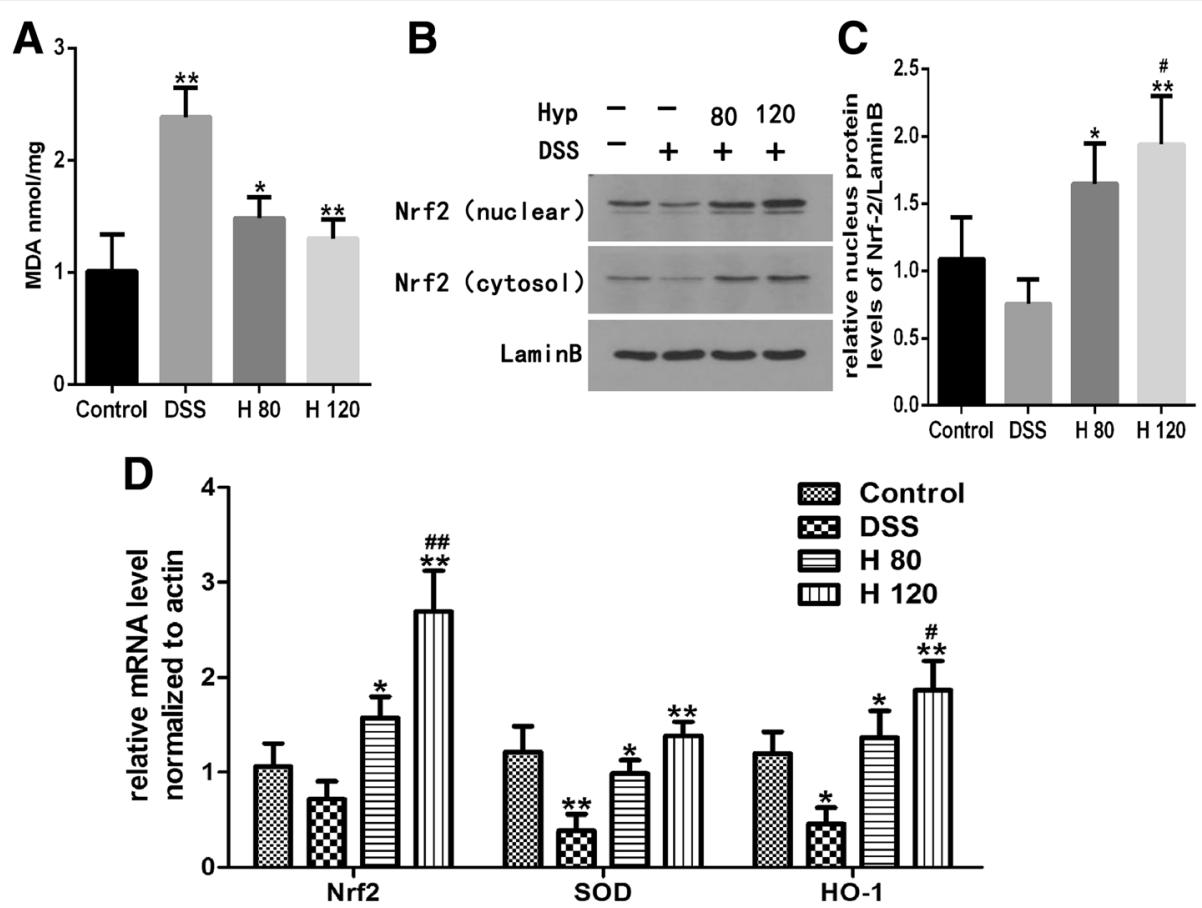

Fig. 6 Hyp reduced colonic levels of MDA and activated Nrf2 pathway. a The levels of MDA. b The expressions of Nrf2 via western blotting. c The relative protein expressions of nucleus Nrf2 were normalized to Lamin B. d Gene expression of Nrf2、SOD and HO-1 via RT-PCR. H 80 and H 120, mice treated with DSS and Hyp 80 and $120 \mathrm{mg} / \mathrm{kg}$ respectively. Data are presented as mean $\pm S . D$. $(n=7)$. Significance: * $P<0.05$, ** $P<0.01$ in comparison with DSS model group; ${ }^{\#} P<0.05, " \# P<0.01$ in comparison with control group

affected patients and likely increases the risk of colorectal cancer [20]. The drugs that are currently used to alleviate the clinical symptoms and inflammation associated with UC include aminosalicylates, corticosteroids, immunosuppressors and biological agents. However, these drugs also have some adverse effects. Therefore, studies seeking to develop safe, effective and targeted therapies for UC are needed. The present study explored the protective effects of Hyp on DSS-induced UC.

The mouse model of DSS-induced colitis is a wellestablished experimental model that shares many signs and symptoms with human UC, including weight loss, diarrhea, emaciation, melena, mucosal ulcerations, colonic shortening and inflammatory cell infiltration [3]. Severe diarrhea, bloody stools, weight loss, and decreases in activity and appetite appeared on the third day after DSS exposure. After the experiment, the DSSinduced group exhibited a remarkably higher DAI score than the healthy control group. We observed DSSinduced pathological changes in the colonic tissue samples by HE staining and found that the mice in the DSS model group displayed prominent abnormalities in the colonic architecture, such as mucosal ulcerations, glandular defects and inflammatory cell infiltration. Moreover, the pathological scores were significantly higher in the DSS model group than in the healthy control group.
Thus, the UC model was successfully established. Based on our results, Hyp alleviates the symptoms and pathological changes in the intestine that are characteristic of experimental UC in mice.

Natural herbal compounds containing Hyp have been proposed to be safer than conventional medicines and are used widely [21]. The administration of 50-200 mg/ $\mathrm{kg}$ Hyp exerted a potent hepatoprotective effect on a mouse model of liver injury by upregulating the Nrf2 pathway $[15,22]$. In addition, Hyp showed certain toxicity during embryonic/fetal development in rats at the dose of $1000 \mathrm{mg} / \mathrm{kg}$ [23]. Based on these findings, our dosing level was safe and efficient. Hyp exerted protective effects on DSS-induced colitis in the present study. However, in other studies, the administration of 10-40 $\mathrm{mg} / \mathrm{kg}$ Hyp significantly suppressed the NF-kB signaling pathway in mouse models of allergic airway inflammation and pancreatic tumors [14, 24]. The effective range of Hyp concentrations seems to be quite different. The discrepancies in the results may be explained by the use of different disease models, the biological and/or interlaboratory variability or by a bell-shaped dose-response curve. Therefore, additional pharmacokinetic and toxicology experiments should be performed with Hyp to further elucidate the mechanism of Hyp before its clinical application. 
Inflammatory cells and the secretion of related inflammatory mediators play critical roles in the development and progression of UC [25]. NF-kB, a key factor in the immune system, regulates the expression of several proinflammatory factors, such as TNF- $\alpha$, IL-4, IL-6, COX-2, adhesion molecule- 1 and chemokines. These factors eventually induce local inflammation and immune dysfunction, changes that trigger a positive feedback loop leading to the development of and increases in inflammation, resulting in intestinal mucosal damage [26]. Those inflammatory mediators are significantly upregulated not only in experimental colitis $[25,27]$ but also in patients with IBD $[28,29]$. Based on accumulating evidence, the inhibition of inflammatory mediators attenuates experimental models of IBD $[14,30]$. IL-10 is an anti-inflammatory factor that plays a protective role in UC. IL-10- and IL10 receptor-deficient mice spontaneously develop colitis and are widely used murine models of IBD [31, 32]. We measured the expression levels of related inflammatory mediators to explore the protective effects of Hyp on colitis. Hyp significantly reduced the levels of TNF- $\alpha$, IL- 6 and COX-2 and increased the levels of IL-10. Thus, Hyp exerts its preventative and therapeutic effects by correcting the imbalance between proinflammatory and anti-inflammatory mediators.

Increased rates of colonic apoptosis have been reported in patients with IBD and experimental colitis $[33,34]$. Increases in the rate of intestinal epithelial cell apoptosis destroy the structure of the epithelial barrier and contribute to the development of intestinal damage [4]. B-cell lymphoma-2 family members, including Bcl-2 and Bax, play a vital role in cell apoptosis. $\mathrm{Bcl}-2$ is an anti-apoptotic protein, whereas Bax is regarded as a proapoptotic protein, as it binds to and antagonizes the effects of $\mathrm{Bcl}-2$ [4]. Increases in the $\mathrm{Bax} / \mathrm{Bcl}-2$ ratio increase the level of cytochrome $\mathrm{c}$ in the cytoplasm by inducing its release from the mitochondria. Cytochrome c activates Caspase-3, one of the major executioner caspases in the apoptosis pathway [4,33]. According to our data, Hyp upregulates the expression of Bcl-2 and downregulates the expression of the pro-apoptotic protein Bax and Caspase-3, indicating that Hyp mitigates colonic apoptosis in the mouse model of DSS-induced colitis. These findings are consistent with those of a report on the inhibitory effects of Hyp on apoptosis in $\mathrm{H}_{2} \mathrm{O}_{2}$ treated human umbilical vein endothelial cells but are inconsistent with those of a report showing that Hyp enhances apoptosis in pancreatic cancer [14]. As shown in the study by Crespo I et al. [33], oxidative stress triggers the expression of several genes linked to apoptotic cell death. The effects of Hyp on colonic apoptosis are likely attributed to its ability to suppress oxidative stress, as epithelial cell apoptosis is enhanced when the intestinal mucosa is exposed to excess ROS levels under inflammatory conditions [35].
Therefore, we assessed the effects of Hyp on the Nrf2 signaling pathway and NF- $\mathrm{KB}$ p65 to elucidate the mechanisms underlying the anti-inflammatory and antiapoptotic effects of Hyp. NF- $\mathrm{kB}$, which plays a vital role in changes in the inflammatory phenotype, triggers the expression of several proinflammatory genes and enhances colonic mucosal inflammation in response to stress [1]. Colonic inflammation is regulated by the NF- $\mathrm{kB}$ pathway [25]. Based on our results, Hyp attenuates the inflammatory response by suppressing NF- $\mathrm{kB}$ activation, consistent with previous studies [30]. Nrf2 is a transcription factor linked to the transactivation of various detoxifying enzymes, and the Nrf2 pathway is known to regulate oxidative stress and the inflammatory response [12]. Kensler [8] confirmed that Nrf2 gene-knockout mice tend to be more susceptible to azoxymethane (AOM)/DSS-induced colitis than wild-type mice due to increases in the levels of inflammatory mediators and reductions in the levels of phase II detoxifying/antioxidant enzymes. Moreover, LPSinduced NF- $\mathrm{kB}$ activation is inhibited by $\mathrm{Nrf} 2$ activation [36]. HO-1, the main antioxidant molecule regulated by Nrf2, plays a critical role in inhibiting oxidative stress processes. The Nrf2 signaling pathway was recently reported to be involved in apoptosis. Activation of the $\mathrm{Nrf} 2 / \mathrm{HO}-1$ pathway attenuates apoptosis by inhibiting Caspase- 8 activation [37], and Nrf2 activation plays a positive role in inducing the expression of anti-apoptosis genes, such as $\mathrm{Bcl} 2$ and Bcl-xl [38]. Uncontrolled ROS surges are a key trigger of mitochondrial dysfunction and mitochondrial breakdown, changes that eventually lead to cell apoptosis. In the present study, Nrf2, a major antioxidant transcription factor, and its downstream antioxidant enzymes, HO1 and SOD, were activated by Hyp. The protective effects of Hyp may be mediated by the activation of the Nrf2 signaling pathway. The major limitation of this study was its lack of evidence supporting the hypothesis that Hyp exerts its beneficial effects by activating the Nrf2 signaling pathway. Therefore, in future studies, we will block the Nrf2 pathway to test this hypothesis.

\section{Conclusions}

Based on the results of the present study, Hyp exerts protective effects on DSS-induced colitis in mice, effects that may be due to the suppression of inflammation and apoptosis via the activation of the Nrf2 signaling pathway, which exerts antioxidant effects.

\section{Abbreviations}

ARE: Antioxidant response element; CMC: Carboxymethyl cellulose; COX2: Cyclooxygenase-2; DAB: Diaminobenzidine; DAl: Disease activity index; DSS: Dextran sodium sulfate; HO-1: Hemeoxygenase-1; Hyp: Hyperoside; IBD: Inflammatory bowel diseases; Keap1: Kelch-like ECH-associated protein 1; MDA: Malondialdehyde; Nrf2: Nuclear factor-erythroid 2-related factor 2; PVDF: Polyvinylidene difluoride; ROS: Reactive oxygen species;

SOD: Superoxide dismutase; TBA: Thiobarbituric acid; UC: Ulcerative colitis 


\section{Acknowledgments}

Not applicable

\section{Funding}

Not applicable

\section{Availability of data and materials}

The datasets of the current study are available from the corresponding author upon reasonable request

\section{Authors' contributions}

$L Y, L S$ and $Y L$ performed the experiments; $L Y$ analyzed the data, prepared the figures and drafted the manuscript; LS, YXL, SJ and SW edited and revised themanuscript; LY and LS interpreted the results of the experiments; $L Y$ is responsible for conception and design of the research. All authors read and approved the final manuscript.

\section{Ethics approval}

All experimental procedures were conducted in conformity with institutional guidelines for the care and use of laboratory animals in Renmin Hospital of Wuhan University, Wuhan, China.

\section{Consent for publication}

Not applicable

\section{Competing interests}

The authors declare that they have no competing interests.

\section{Publisher's Note}

Springer Nature remains neutral with regard to jurisdictional claims in published maps and institutional affiliations.

Received: 25 May 2017 Accepted: 6 November 2017

Published online: 14 November 2017

\section{References}

1. Biasi F, Leonarduzzi G, Oteiza Pl, et al. Inflammatory bowel disease: mechanisms, redox considerations, and therapeutic targets. Antioxidants \& Redox Signaling. 2013;19:1711.

2. Sánchezfidalgo S, Cárdeno A, Sánchezhidalgo M, et al. Dietary extra virgin olive oil polyphenols supplementation modulates DSS-induced chronic colitis in mice. Journal of Nutritional Biochemistry. 2013;24:1401-13.

3. Chiou YS, Ma JL, Sang S, et al. Peracetylated (-)-Epigallocatechin-3-gallate (AcEGCG) Potently Suppresses Dextran Sulfate Sodium-Induced Colitis and Colon Tumorigenesis in Mice. Journal of Agricultural \& Food Chemistry. 2012;60:3441-51

4. Becker C, Watson AJ, Neurath MF. Complex roles of caspases in the pathogenesis of inflammatory bowel disease. Gastroenterology. 2013;144: 283-93.

5. Pandurangan AK, Mohebali N, Norhaizan ME, et al. Gallic acid attenuates dextran sulfate sodium-induced experimental colitis in BALB/C mice. Drug Design Development \& Therapy. 2015;9:3923-34

6. Bryan HK, Olayanju A, Goldring CE, et al. The Nrf2 cell defence pathway: Keap1-dependent and -independent mechanisms of regulation. Biochemical Pharmacology. 2013;85:705-17.

7. Uruno A, Motohashi $\mathrm{H}$. The Keap1-Nrf2 system as an in vivo sensor for electrophiles. Nitric Oxide Biology \& Chemistry. 2011;25:153-60.

8. Osburn WO, Karim B, Dolan PM, et al. Increased colonic inflammatory injury and formation of aberrant crypt foci in Nrf2-deficient mice upon dextran sulfate treatment. International Journal of Cancer. 2007:121:1883-91.

9. Khor TO, Huang MT, Prawan A, et al. Increased susceptibility of Nrf2 knockout mice to colitis-associated colorectal cancer. Cancer Prevention Research. 2008:1:187-91.

10. Wang $Y$, Wang H, Qian C, et al. 3-Aroylmethylene-2,3,6,7-tetrahydro-1Hpyrazino[2,1-a]isoquinolin-4(11bH)-ons (compound 1), a novel potent Nrf2/ ARE inducer, protects against DSS-induced colitis via inhibiting NLRP3 inflammasome. Biochemical Pharmacology. 2016;101:71-86.

11. Yue L, Lei S, Luo H. Luteolin ameliorates dextran sulfate sodium-induced colitis in mice possibly through activation of the Nrf2 signaling pathway. International Immunopharmacology. 2016;40:24.
12. Kim J, Cha YN, Surh YJ. A protective role of nuclear factor-erythroid 2related factor-2 (Nrf2) in inflammatory disorders. Mutation Research. 2010; 690(1-2):12-23

13. $\mathrm{Xu} \mathrm{D}$, Chen $\mathrm{L}$, Chen $\mathrm{X}$, et al. The triterpenoid CDDO-imidazolide ameliorates mouse liver ischemia-reperfusion injury through activating the Nrf2/HO-1 pathway enhanced autophagy. Cell Death \& Disease. 2017;8(8):e2983.

14. Li Y, Wang Y, Li L, et al. Hyperoside induces apoptosis and inhibits growth in pancreatic cancer via $\mathrm{BCl}-2$ family and NF-KB signaling pathway both in vitro and in vivo. Tumor Biology. 2016;37:7345-55.

15. Choi JH, Kim DW, Yun N. Protective effects of hyperoside against carbon tetrachloride -induced liver damage in mice. J Nat Prod. 2011;74:1055.

16. Xing HY, Liu Y, Chen JH. Hyperoside attenuates hydrogen peroxide-induced L02 cell damage via MAPK-dependent Keap1 -Nrf2 -ARE signaling pathway. Biochem Biophys Res Commun. 2011:410:759.

17. Li ZL, Liu JC, Hu J, et al. Protective effects of hyperoside against human umbilical vein endothelial cell damage induced by hydrogen peroxide. Journal of Ethnopharmacology. 2012;139:388-94.

18. Ito R, Shin-Ya M, Kishida T, et al. Interferon-gamma is causativelyinvolved in experimental inflammatory bowel disease in mice. Clin Exp Immunol. 2006; 146:330-8.

19. Dieleman LA, Palmen MJ, Akol H, et al. Chronic experimental colitis induced by dextran sulphate sodium (DSS) is characterized by Th1 and Th2 cytokines. Clinical \& Experimental Immunology. 1998;114:385-91.

20. Pandurangan AK, Esa NM. Signal transducer and activator of transcription 3 - a promising target in colitis-associated cancer. Asian Pacific Journal of Cancer Prevention Apjcp. 2014;15:551-60.

21. Ekor $M$. The growing use of herbal medicines: issues relating to adverse reactions and challenges in monitoring safety. Frontiers in Pharmacology. 2014:4(4):177.

22. Xie W, Jiang Z, Wang J, et al. Protective effect of hyperoside against acetaminophen (APAP) induced liver injury through enhancement of APAP clearance. Chemico-biological interactions. 2016;246(March):11.

23. Ai G, Huang Z, Wang D, et al. Study on toxicity of hyperoside in rat embryo-fetal development. China Journal of Chinese Materia Medica. 2012:37(16):2452

24. Ye $\mathrm{P}$, Yang $\mathrm{XL}$, Chen $\mathrm{X}$, et al. Hyperoside attenuates OVA-induced allergic airway inflammation by activating Nrf2. International Immunopharmacology. 2017:44:168-73.

25. Wang H, Gu J, Hou X, et al. Anti-inflammatory effect of miltirone on inflammatory bowel disease via TLR4/NF-KB/IQGAP2 signaling pathway. Biomedicine \& Pharmacotherapy. 2017:85:531-40.

26. Siddique I, Khan I. Mechanism of regulation of $\mathrm{Na}-\mathrm{H}$ exchanger in inflammatory bowel disease: role of TLR-4 signaling mechanism. Digestive Diseases and Sciences. 2011:56:1656-62.

27. Park SY, Neupane GP, Lee SO, et al. Protective effects of Pogostemon cablin Bentham water extract on inflammatory cytokine expression in TNBS-induced colitis in rats. Archives of Pharmacal Research. 2014;37: 253-62.

28. Roberts PJ. Neuronal COX-2 expression in human myenteric plexus in active inflammatory bowel disease. Gut. 2001;48:468-72.

29. Autschbach F, Giese T, Gassler N, et al. Cytokine/chemokine messenger-RNA expression profiles in ulcerative colitis and Crohn's disease. Virchows Archiv An International Journal of Pathology. 2002;441(5):500.

30. Ferrari $D$, Speciale A, Cristani $M$, et al. Cyanidin-3-O-glucoside inhibits NF-kB signalling in intestinal epithelial cells exposed to TNF-a and exerts protective effects via Nif2 pathway activation. Toxicology Letters. 2016;264:51-8.

31. Hart ML, Aaron C. Ericsson, et al. Differing Complex Microbiota Alter Disease Severity of the IL-10-/- Mouse Model of Inflammatory Bowel Disease. Frontiers in. Microbiology. 2017;8:792.

32. Shouval DS, Biswas A, Kang $Y H$, et al. Interleukin $1 \beta$ Mediates Intestinal Inflammation in Mice and Patients With Interleukin 10 Receptor Deficiency. Gastroenterology. 2016;151:1100

33. Crespo I, Sanmiguel B, Prause C, et al. Glutamine Treatment Attenuates Endoplasmic Reticulum Stress and Apoptosis in TNBS-Induced Colitis. Plos One. 2012;7:e50407.

34. Blander JM. Death in the intestinal epithelium - Basic biology and implications for inflammatory bowel disease. Febs Journal. 2016;283(14):2720-30.

35. Kruidenier L, Kuiper I, Lamers CB, et al. Intestinal oxidative damage in inflammatory bowel disease: semi-quantification, localization, and association with mucosal antioxidants. The Journal of pathology. 2003;201:28-36. 
36. Lin W, Wu RT, Wu T, et al. Sulforaphane suppressed LPS-induced inflammation in mouse peritoneal macrophages through Nrf2 dependent pathway. Biochemical Pharmacology. 2008;76(8):967-73.

37. Wan $P$, Su W, Zhang $Y$, et al. Trimetazidine protects retinal ganglion cells from acute glaucoma via the Nrf2/Ho-1 pathway. Clinical Science. 2017;131: 2363-75.

38. Young-Ok S, Poyil P, Ram Vinod R, et al. Antioncogenic and Oncogenic Properties of Nrf2 in Arsenic-induced Carcinogenesis. Journal of Biological Chemistry. 2015;290(45):27090-100.

Submit your next manuscript to BioMed Central and we will help you at every step:

- We accept pre-submission inquiries

- Our selector tool helps you to find the most relevant journal

- We provide round the clock customer support

- Convenient online submission

- Thorough peer review

- Inclusion in PubMed and all major indexing services

- Maximum visibility for your research

Submit your manuscript at www.biomedcentral.com/submit 